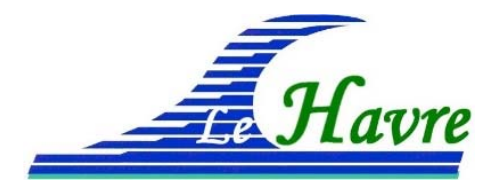

XVI èmes Journées Nationales Génie Côtier - Génie Civil

Le Havre, 2020

DOI:10.5150/jngcgc.2020.022 ～(C) Editions Paralia CFL

disponible en ligne - http://www.paralia.fr - available online

\title{
Intercomparaison de modèles morphodynamiques sur la plage du Truc Vert - campagne ECORS' 2008
}

\author{
Julien CHAUCHAT ${ }^{1}$, Gael Lö̈c RICHARD ${ }^{2}$, Cyrille BONAMY ${ }^{1}$, \\ Kiet NGUYEN ${ }^{1}$, Hassan SHAFIEI ${ }^{1}$
}

\author{
1. Univ. Grenoble Alpes, CNRS, Grenoble INP, LEGI, 38000 Grenoble, France. \\ Institute of Engineering Univ. Grenoble Alpes, France. \\ Julien.Chauchat@univ-grenoble-alpes.fr \\ 2. Univ. Grenoble Alpes, INRAE, ETNA, 38000 Grenoble, France.
}

\section{Résumé :}

La simulation morphodynamique des plages sableuses est un problème complexe couplant l'hydrodynamique des courants (marée, dérive littoral), la propagation des vagues et leurs déferlements au transport sédimentaires conduisant à l'évolution morphologique de la plage. Plusieurs systèmes de modélisation numérique sont disponibles pour réaliser de telles simulations, dans le cadre de l'étude amont MEPELS pilotée par le SHOM nous proposons une intercomparaison de trois modèles opensource: CROCO, Delft3D et Xbeach. Le cas d'application choisi est la campagne ECORS' 2008 menée sur la plage du Truc Vert en aquitaine (SENECHAL et al., 2011). Durant cette campagne, 4 tempêtes ont frappé le site d'étude dont l'une de période de retour 10 ans $(\mathrm{Hs} \sim 8 \mathrm{~m})$ et la morphologie de la plage a beaucoup évolué. Deux bathymétries sont disponibles, la première du 11 février 2008 et la seconde du 4 avril 2008. Ces deux bathymétries sont utilisées pour initialiser et quantifier la performance des différents modèles respectivement. Dans un premier temps, l'hydrodynamique des trois modèles est calibrée sur les mesures de la période calme du 6 avril au 8 avril 2008. Dans un second temps, des simulations morphodynamiques sont réalisées avec chacun de ces modèles sur la période du 11 février au 4 avril en prenant tous les paramètres d'entrée pour le sédiment par défaut. Pour l'instant, seule la simulation avec CROCO a été réalisée. L'analyse de cette première simulation montre une évolution morphologique importante de la plage ... Une analyse plus approfondie de la simulation concernant la migration des barres interne et externe reste à mener et à comparer avec les mesure vidéo de ALMAR et al (2010). Une étude de sensibilité aux paramètres de déferlement et aux paramétrisations de transport de sédiments sera réalisée. Par la suite, la même méthodologie sera employée avec Xbeach et Delft3D, la qualité des simulations pour les différents modèles sera quantifiée par des indicateurs objectifs (Brier Skill Score et erreur).

\section{Mots-clés :}

Morphodynamique des plages sableuses, Processus sédimentaires, Simulation numérique 


\section{Thème 2 - Dynamique sédimentaire}

\section{Introduction}

Le travail présenté dans cet article s'inscrit dans le cadre du projet stratégique MEPELS (Modèle d'Evolution des Plages et Environnements Littoraux Sableux) piloté par le SHOM (Service Hydrographique et Océanographique de la Marine). La présente étude a pour principal objet de faire un état des lieux des modèles candidats pour le système MEPELS et de proposer une sélection de ces modèles sur des critères objectifs. Elle doit évaluer les processus modélisés et les avantages et apports de chacun des modèles, afin de définir la liste des modèles à intégrer à terme dans le système.

Suite à une étude bibliographique, les trois modèles numériques suivants ont été retenus : CROCO (WARNER et al., 2008), DELFT3D (LESSER et al., 2004) et XBEACH (ROELVINK et al., 2009). Tous ces modèles ont une reconnaissance internationale démontrée, sont libre de droit quant à l'accès aux codes sources et possèdent des spécificités particulières qui les rendent attractifs au regard des objectifs du projet MEPELS.

Le système CROCO présente des caractéristiques numériques avancées avec la possibilité d'utiliser des schémas numériques d'ordre élevés (5ème ordre en espace) et un modèle à pression non-hydrostatique efficient. Le modèle DELFT3D possède une interface Matlab très développée pour les applications côtières et un grand nombre de paramétrisations pour la propagation des vagues et les flux de sédiments. Le modèle XBEACH en mode surfbeat permet de simuler les ondes infragravitaires qui sont essentielles pour simuler des événements extrêmes de tempêtes et il dispose également d'un mode nonhydrostatique permettant de faire des simulations vagues-à-vagues.

Dans cet article nous présentons les résultats préliminaires de l'application de ces trois modèles à la campagne ECORS' 2008 sur la plage du Truc Vert (SENECHAL et al., 2011). La campagne ECORS est présentée dans la section 2. Dans la section 3, la calibration hydrodynamique des modèles est présentée. Les résultats des premières simulations morphodynamiques sur la période du 11 février au 4 avril sont présentés dans la section 4.

\section{La campagne terrain ECORS'2008 - plage du Truc Vert}

Lors de la campagne ECORS'2008 sur la plage de Truc Vert, côte sud-ouest de la France, les caractéristiques des vagues ont été mesurées à 20 mètres de profondeur. Les données mesurées (filtrées) sont représentées sur la figure 1 pour la hauteur significative des vagues (Hs), la période de pic spectrale des vagues (Tp) et la direction des vagues (prise perpendiculaire à la plage). Le signal de marée fourni par le SHOM est également indiqué dans le panneau supérieur. Le panneau du bas montre le nombre de Dean De $=\mathrm{Hs} / \mathrm{Tp}$ Ws, où Ws est la vitesse de chute des sédiments. Une valeur de $\mathrm{Ws}=3,3 \mathrm{~cm} / \mathrm{s}$ est utilisée correspondant à des sables moyens $(\mathrm{d} 50=300 \mu \mathrm{m})$. 

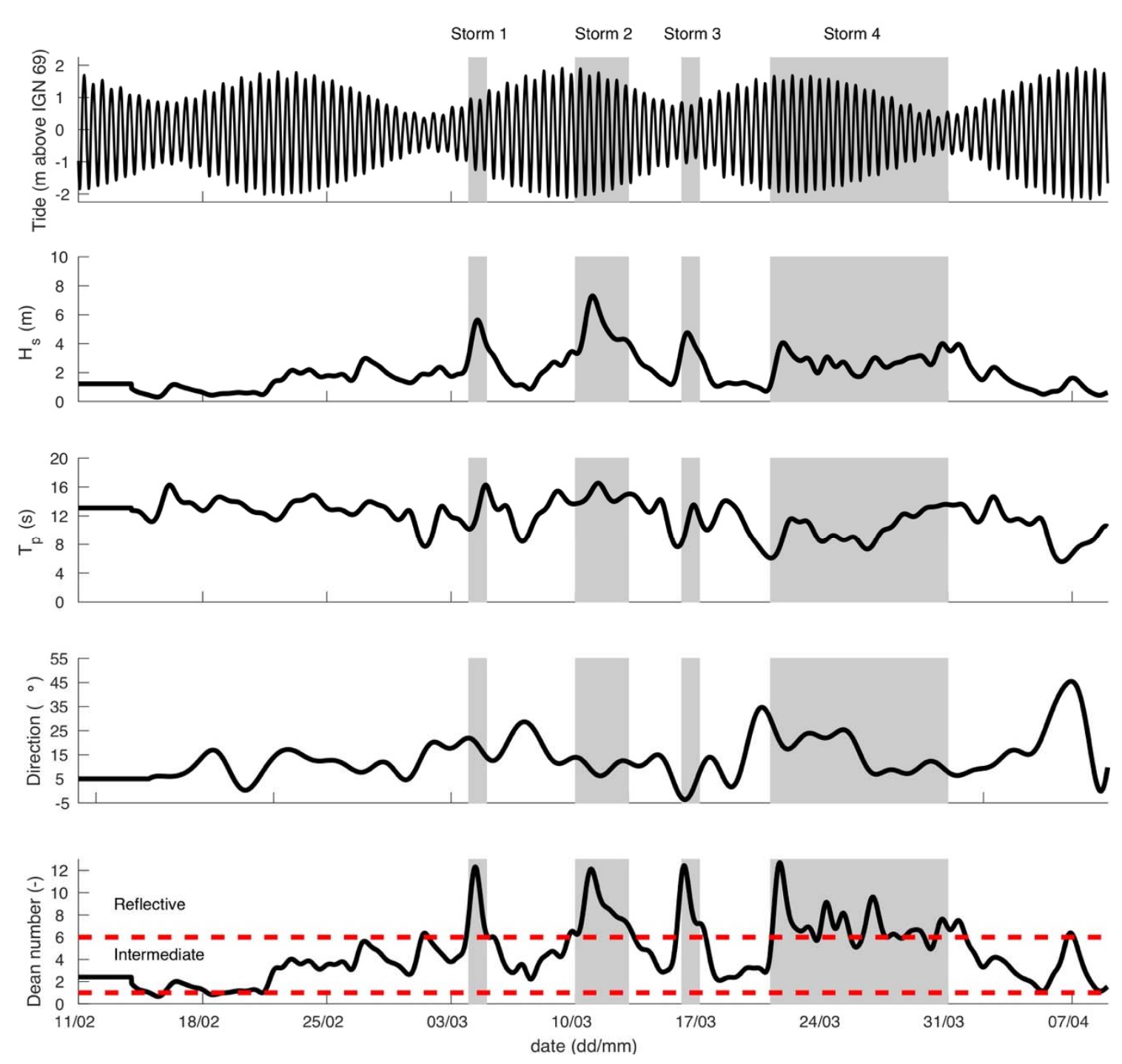

Figure 1. Série temporelle de marée, hauteur significative des vagues (Hs), période de pic spectral (Tp), direction des vagues $\left(^{\circ}\right)$ et de nombre de Dean (De=Hs/Ws Tp) à $20 \mathrm{~m}$ de profondeur au large de la plage du Truc Vert Beach pendant la campagne ECORS'2008 (d'après SENECHAL et al., 2011).

La série temporelle montre l'occurrence de 4 tempêtes, la première a duré un jour, le 5 mars, avec $\mathrm{Hs}<4 \mathrm{~m}, \mathrm{Tp}=14 \mathrm{~s}$ et un angle d'incidence des vagues modéré $\left(\sim 8^{\circ} \mathrm{W}-\mathrm{NW}\right)$. Pendant cette épisode, l'amplitude de marée était intermédiaire $\sim 3 \mathrm{~m}$. La deuxième tempête qui a frappé la plage du Truc Vert a été sévère avec Hs d'environ $8 \mathrm{~m}$, une période de pointe de $\mathrm{Tp}=18 \mathrm{~s}$ et un angle d'incidence de $15^{\circ}(\mathrm{W}-\mathrm{NW})$ et une amplitude de marée printanière $(\sim 4 \mathrm{~m})$. La tempête 2 a duré 3 jours. La troisième tempête a eu lieu du $16 \mathrm{au}$ 17 mars, la hauteur de vague significative était de $\mathrm{Hs}=6 \mathrm{~m}$ avec une période de pic $\mathrm{Tp}=12 \mathrm{~s}$ et une incidence presque normale à la plage. L'amplitude des marées était d'environ 2,5 m. Après une période de 5 jours de conditions de basse énergie, la quatrième 


\section{Thème 2 - Dynamique sédimentaire}

tempête a atteint la plage du Truc Vert avec Hs $>3$ m et $\mathrm{Tp}>12 \mathrm{~s}$ et a duré 10 jours du 21 au 31 mars avec une forte incidence des vagues $\left(15-20^{\circ}\right)$. Au cours de cette tempête, l'amplitude des marées a varié de vives eaux $(3,8 \mathrm{~m})$ à mortes eaux $(1,5 \mathrm{~m})$.

La bathymétrie initiale et finale de la plage du Truc Vert pendant la campagne de terrain ECORS 2008 est présentée dans la figure 2. À l'aide d'une technique d'imagerie vidéo, ALMAR et al. (2010) ont pu déduire l'évolution morphologique des barres externes et internes sous l'influence de ces quatre tempêtes. La barre externe a surtout évolué au cours de la deuxième tempête, passant d'une forme en croissant vers une forme plus uniforme le long de la côte accompagnée d'une migration vers le large d'environ $100 \mathrm{~m}$ Au cours de la quatrième tempête, des motifs en croissants se sont développé sur la barre externe accompagné d'un épaississement dans la direction transverse.

\section{Validation hydrodynamique}

Dans un premier temps, une validation hydrodynamique des 3 modèles numériques, CROCO, Delft3D et Xbeach, est réalisée. Cette validation porte sur la période du 6 au 8 avril 2008 pendant laquelle 2 capteurs de vitesse et de hauteurs de vagues ont été déployé dans la zone intertidale : VEC1 et VEC3 (voir figure 3 pour la localisation).

Dans les simulations présentées ci-après, les paramètres ont été fixés à leurs valeurs par défaut pour tous les modèles. Le tableau 1 recense les principaux paramètres clefs des modèles et les paramétrisations retenues.

Tableau 1. Synthèse des indicateurs objectifs de qualité des simulations morphologiques.

\begin{tabular}{llllll}
\hline Modèles & frott. courant & frott. vague & gamma & alpha & beta \\
\hline CROCO & $z 0=5 \mathrm{~mm}$ & SOULSBY (1995) & 0.3 & 0.5 & 0.1 \\
Delft3D & Manning $n=0.02$ & COLLINS (1972) & 0.6 & 1 & 0.1 \\
XBEACH & $z 0=5 \mathrm{~mm}$ & fw $=0.15$ & 0.78 & 1 & 0.1 \\
\hline
\end{tabular}

La figure 2 montre la comparaison des hauteurs de vagues (panneau du haut), de la vitesse transversale (cross-shore, panneau du milieu) et le long de la plage (long-shore, panneau du bas) pour VEC1 (à gauche) et VEC3 (à droite). La simulation Delft3D n'a été réalisée que sur 2 cycles de marées pour l'instant. Dans l'ensemble les résultats obtenus avec ces trois modèles sont satisfaisant, l'atténuation des vagues entre la frontière au large et les points VEC1 et VEC3 sont bien reproduites bien qu'une légère sous-estimation de l'atténuation soit observée, en particulier pour CROCO.

Concernant les courants transverses, les modèles CROCO et Xbeach reproduisent très bien l'amplitude et la phase de ces courants pour les deux points de mesures. Delft3D présente des oscillations importantes et sous-estime l'amplitude de ces courants. Concernant la composante le long de la plage, Delft3D donne de très bons résultats alors que CROCO surestime sensiblement cette composante et plus particulièrement pendant 


\section{XVIèmes Journées Nationales Génie Côtier - Génie Civil \\ Le Havre 2020}

la marée haute du 7 avril. Le modèle Xbeach est nettement moins bon que les deux autres modèles avec des courants trop intenses durant les marées hautes sur l'ensemble de la période. Une étude de sensibilité a été réalisée mais elle n'a pas permis d'améliorer ces résultats pour l'instant. A ce stade, il semble que la paramétrisation du modèle de roller soit la clef pour reproduire fidèlement ces mesures et plus particulièrement le breaker delay introduit dans Delft3D et utilisé dans la simulation présentée sur la figure 2.
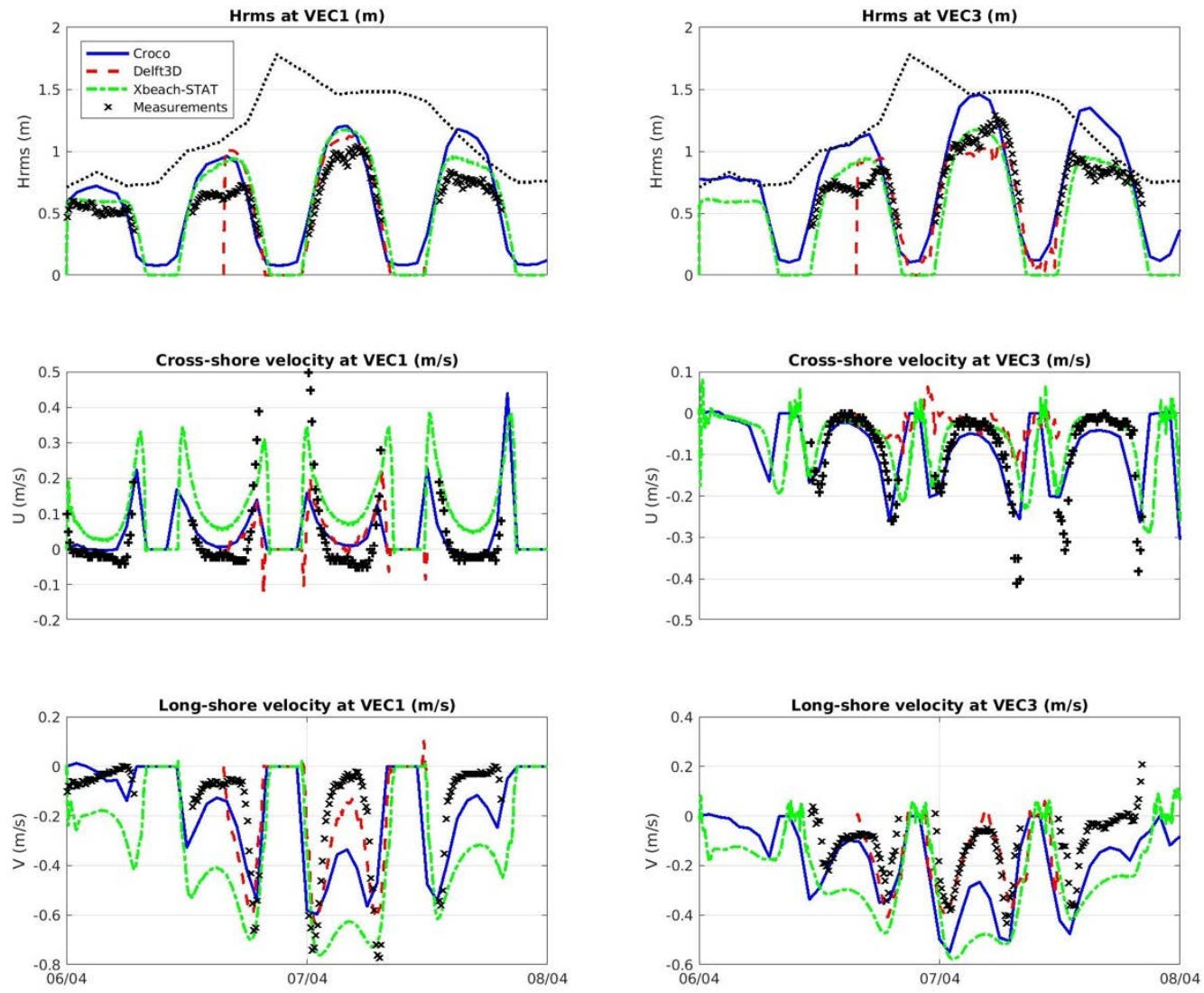

Figure 2. Validation hydrodynamique des 3 codes de calculs : CROCO, Delft3D, Xbeach sur les mesures VEC1 et VEC3 pendant la période du 6 au 8 avril 2008.

\section{Simulation morphodynamique de la campagne ECORS'2008}

Dans l'objectif de quantifier de la manière objective la qualité des simulations morphodynamiques, le Brier Skill Score (BSS) ou Mean-Squared Error Skill Score, est utilisé, il est défini de la manière suivante :

$B S S=1-\left\langle(p-o)^{2}\right\rangle /\left\langle(r-o)^{2}\right\rangle$

où $\mathrm{p}$ représente la bathymétrie prédite, o la bathymétrie observée et $\mathrm{r}$ la bathymétrie initiale. \langle\rangle représente un opérateur de moyenne spatiale sur le domaine de calcul. Le BSS représente la façon dont le modèle prédit la bathymétrie par rapport à la bathymétrie initiale. Si la prédiction du modèle est égale au profil initial (rien ne se passe selon le modèle), le score de compétence est 0 . Si la prédiction à un instant donnée est égale à la 


\section{Thème 2 - Dynamique sédimentaire}

mesure à ce même instant, la compétence du modèle est parfaite et le score vaut 1 . Lorsque la prédiction du modèle est pire que le profil initial, le score devient négatif. La classification suivante a été donnée par VAN RIJN et al. (2003) : $\mathrm{BSS}<0=$ mauvais, $0<\mathrm{BSS}<0,3=$ pauvre $; 0,3<\mathrm{BSS}<0,6=$ raisonnable $; 0,6<\mathrm{BSS}<0,8=$ bon $; 0,8<\mathrm{BSS}<1=$ excellent.
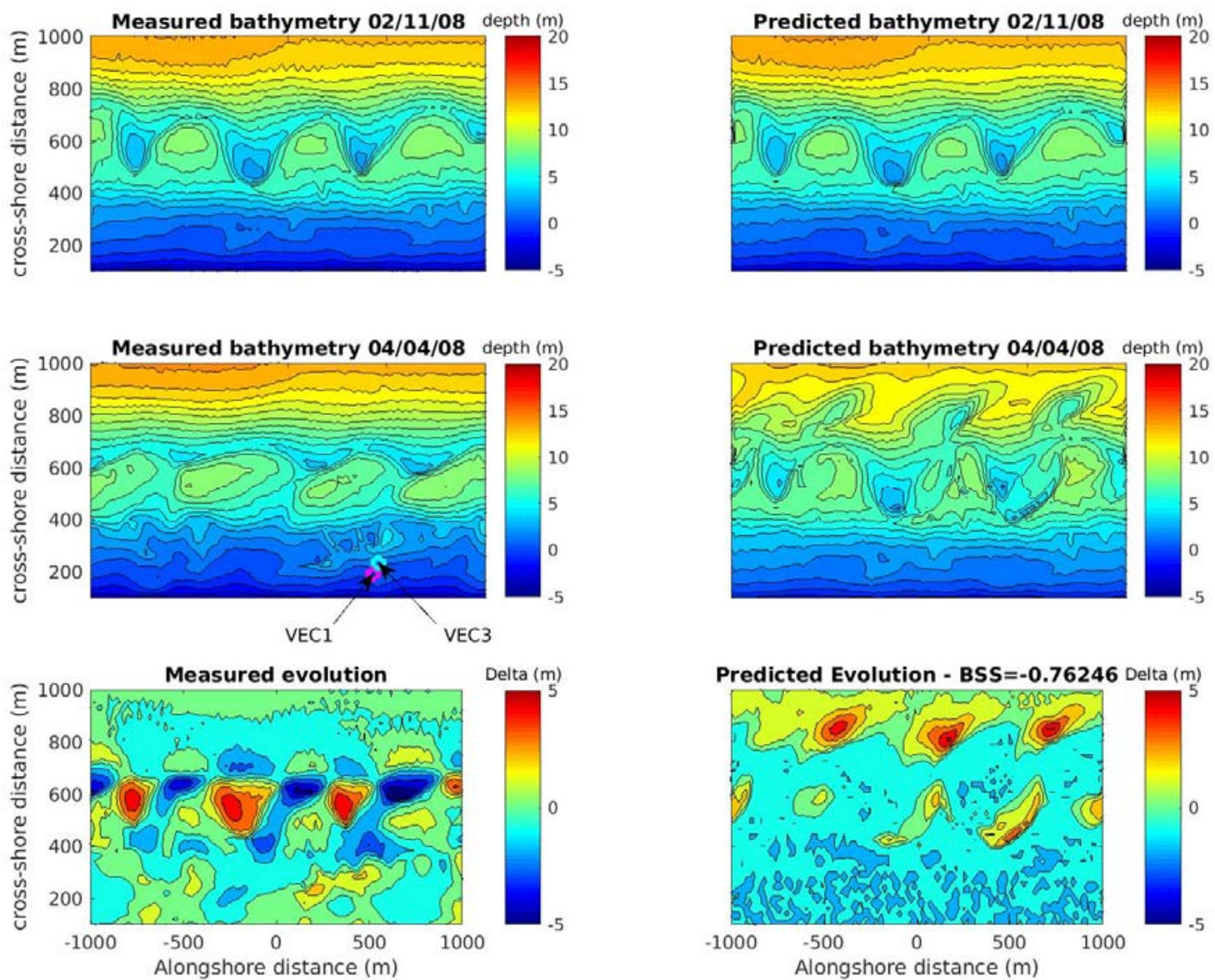

Figure 3. Evolution morphologique mesurée (à gauche) et prédite par CROCO (à droite) pour la période du 11 février au 4 avril 2008. Simulation CROCO à 20m de résolution avec paramètres par défaut.

Les résultats des simulations préliminaires réalisées avec CROCO $(20 \mathrm{~m}$ de résolution, charriage et suspension) sont présentés sur la figure 3. Les panneaux de gauche montrent les mesures et les panneaux de droite le résultat de la simulation sur la période du 11 février au 4 avril 2008 soit 53 jours. Le modèle de charriage est celui proposé par SOULSBY et DAMGAARD (2005) et le module d'érosion est imposé à $E=10^{-2} \mathrm{~kg} / \mathrm{m}^{2} / \mathrm{s}$. Comme en témoigne le $\mathrm{BSS}=-0.76$ (voir tableau 2) la prédiction n'est pas bonne, une deuxième barre externe est prédite au large et les variations de morphologie ne se produisent pas aux bons endroits. Une première étude de sensibilité a été menée (voir 


\section{XVIèmes Journées Nationales Génie Côtier - Génie Civil \\ Le Havre 2020}

tableau 2) en négligeant le charriage (BSS $=-0.12$ ) et en divisant le taux d'érosion par 10 (BSS=0.034). Les résultats sont améliorés lorsque le taux d'érosion est réduit, il faut donc analyser plus en détail le modèle de charriage et tester les paramètres empiriques de ce modèle.

Une simulation morphodynamique a été réalisée avec le code $\mathrm{XBEACH}$ en prenant tous les paramètres sédimentaires par défaut. Le résultat est présenté sur la figure 4 et le BSS est de 0.11 . La prédiction morphologique semble plus raisonnable que dans la simulation CROCO par contre la barre externe a pratiquement complètement disparu. Il faut rappeler que dans la partie calibration hydrodynamique qu'Xbeach a tendance à surestimer les courants le long de la plage ce qui peut expliquer la morphologie prédite par ce modèle. Aucune étude de sensibilité n'a été menée pour l'instant, cela fait partie des perspectives à court terme.
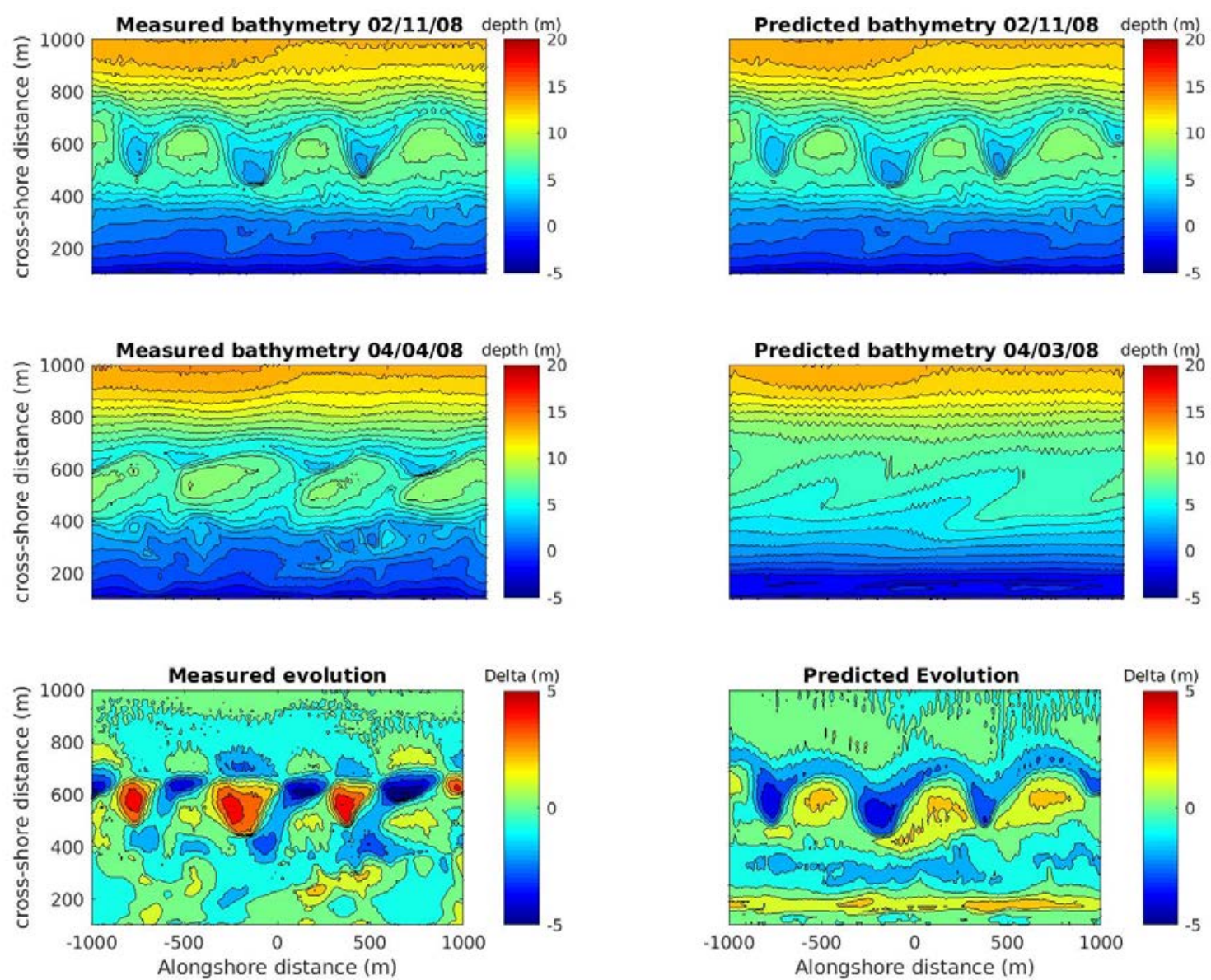

Figure 4. Evolution morphologique mesurée (à gauche) et prédite par XBEACH (à droite) pour la période du 11 février au 4 avril 2008. Simulation XBEACH à 10m de résolution avec paramètres par défaut. 


\section{Thème 2 - Dynamique sédimentaire}

Tableau 2. Synthèse des indicateurs objectifs de qualité des simulations morphologiques.

\begin{tabular}{lll}
\hline Modèles & BSS & Qualité \\
\hline CROCO (20m default) & -0.760 & mauvaise \\
CROCO (sans charriage) & -0.125 & mauvaise \\
CROCO (sans charriage + E/10) & 0.034 & pauvre \\
XBEACH (10m default) & 0.11 & pauvre \\
\hline
\end{tabular}

\section{Conclusions}

En conclusion, dans cet article, nous avons présenté les résultats préliminaires de validation hydrodynamique et de simulation morphologique avec les codes de calculs CROCO, Xbeach et Delft3D. Concernant la calibration hydrodynamique, les résultats obtenus avec CROCO et Delft3D sont très satisfaisant pour les vagues et les courants cross-shore et long-shore. La simulation Xbeach, par contre, surestime les courants longshore. Concernant les simulations morphodynamiques, en prenant tous les paramètres par défaut, il semblerait que Xbeach donne de meilleurs résultats que CROCO bien qu'à ce stade les deux codes ont un score de performance morphologique qualifié de "pauvre" au sens du BSS. En perspective, nous allons évaluer le modèle Delft3D sur cette configuration et réaliser une étude de sensibilité aux paramètres du module sédimentaire pour les 3 codes de calculs.

\section{Références bibliographiques}

ALMAR R., CASTELlE B., RUESSINK B., SENECHAL N., BONNETON P., MARIEU V. (2010). Two- and three-dimensional double-sandbar system behaviour under intense wave forcing and a meso-macro tidal range. Continental Shelf Research, Vol. 30(7), pp 781-792. https://doi.org/10.1016/j.csr.2010.02.001

COLLINS, J., (1972). Prediction of shallow water spectra. Journal of Geophysical Research, Vol. 77 (15) pp 2693-2707. https://doi.org/10.1029/JC077i015p02693

LESSER G., ROELVINK J., VAN KESTER J., STELLING G. (2004). Development and validation of a three-dimensional morphological model. Coastal Engineering, Vol. 51(8-9), pp 883-915. https://doi.org/10.1016/j.coastaleng.2004.07.014

ROELVINK D., RENIERS A., VAN DONGEREN A., VAN THIEL DE VRIES J., MCCALL R., LESCINSKI J. (2009). Modelling storm impacts on beaches, dunes and barrier islands. Coastal Engineering, Vol. 56(11-12), pp 1133-1152. https://doi.org/10.1016/j.coastaleng.2009.08.006 


\section{XVIèmes Journées Nationales Génie Côtier - Génie Civil \\ Le Havre 2020}

SENECHAL N., STÉPHANE A., GALLAGHER E., MACMAHAN J., MASSELINK G., MICHALLET H., RENIERS A., RUESSINK G., RUSSELL P., SOUS D., TURNER I., ARDHUIN F., BONNETON P., BUJAN S., CAPO S., CERTAIN R., PEDREROS R., GARLAN T. (2011). The ECORS-Truc Vert'08 nearshore field experiment: Presentation of a three-dimensional morphologic system in a macro-tidal environment during consecutive extreme storm conditions. Ocean Dynamics, Vol. 61, pp 2073-2098. https://doi.org/10.1007/s10236-011-0472-x

SOULSBY R.L. (1995). Bed shear-stresses due to combined waves and currents. Advances in Coastal Morphodynamics, Stive M., Fredsøe J., Hamm L., Soulsby R., Teisson C., Winterwerp J. (Eds.), Delft Hydraulics, Delft, The Netherlands, pp 420-423. SOULSBY R.L., DAMGAARD J.S. (2005). Bedload sediment transport in coastal waters. Coastal Engineering, Vol. 52 (8), pp 673-689. https://doi.org/10.1016/j.coastaleng.2005.04.003

VAN RIJN L., WALSTRA D., GRASMEIJER B., SUTHERLAND J., PAN S., SIERRA J. (2003). The predictability of cross-shore bed evolution of sandy beaches at the time scale of storms and seasons using process-based profile models. Coastal Engineering, 47(3), pp 295 - 327. https://doi.org/10.1016/S0378-3839(02)00120-5

WARNER J.C., SHERWOOD C.R., SIGNELL R.P., HARRIS C.K., ARANGO H.G. (2008). Development of a three-dimensional, regional, coupled wave, current, and sediment-transport model. Computers \& Geosciences, Vol. 34(10), pp 1284-1306. https://doi.org/10.1016/j.cageo.2008.02.012 
Thème 2 - Dynamique sédimentaire 